\title{
FRANCESCO ILORIG(iO)
}

\section{EMIGRAZIONEE ITAIJINISTICA}

Mi occupo di italo-canadiana da qualche tempo, forse, credo di poter sostenere, almeno per quanto riguarda la dimensione letteraria, da sempre o fin dal suo inizio. Sono stato uno dei pochi recensori di Roman Candles, l'antologia di poesic che Pier Giorgio Di Cicco diede alle stampe nel 1978 e a cui generalmente si fa risalire la nascita ufficiale della letteratura italocanadese. Ho anche creduto subito nel valore didattico delle opere di autori come Mary Di Michele, Mary Melfi, Caterina Edwards, Marco Nicone o Antonio D'Nlfonso o Frank Paci o Nino Ricci o lo stesso Di Cicco. Nell'ex-dipartimento di italiano della Carleton University (Ottawa), ho insegnato, a partire dalla metà degli anni ottanta, da solo prima e con William Anselmi dopo, quello che è stato a lungo nel Canada l'unico corso universitario dedicato a queste opere e a questi autori.

Il mio interesse, però, devo anche subito precisare, non si riconnette se non secondariamente a stimoli o a pressioni di natura istituzionale, a questioni di carriera. Nessuno nella mia università mi ha mai incoraggiato ad insegnare letteratura e cultura italo-canadese. $\mathrm{Al}$ contrario: io e poi William Anselmi abbiamo doruto sempre -quotidianamente- sia opporci alla totale indifferenza della nostra facoltà verso gli aspetti pedagogici o socio-culturali delle cosiddette lingue e/o letterature straniere, sia schivare i sospetti dei colleghi all'interno del dipartimento in cui insegnavamo. Lidea che potesse essere auspicabile introdurre nel nostro programma di laurea testi di poeti o romanzieri o drammaturghi che abitavano fuori d'Italia, non ancora affermati, e che per di più scriverano in inglese $O$ in francese, avera quindici o dieci anni fa, nelle riunioni dipartimentali, tutta l'aria di una provocazione. Ci è voluta una forte dose di pazienza e di testardaggine per riuscire a convincere persone e comitati, nonché gli addetti ai lavori nostri dirimpettai o in altri uffici del nostro corridoio. Idem come sopra, e forse peggio, poi, nelle associazioni professionali. I.o spazio che si trovara nei convegni è stato, fino a poco tempo fa, abbastanza marginale, e comunyue più generoso e confortevole in quelli delle altre discipline che in quelli dell' italianistica. 
No, ciò che mi ha spinto a seguire gli autori e a leggere le loro opere è stato fin dal primo giorno qualcosa di molto diverso. Semplicemente, le poesie, i romanzi, il teatro de me fabula narratur, mi parlavano del mondo in cui vivero. E di questa loro presa sul vissuto - vissuto mio ma anche di una grossa fetta degli studenti a cui insegnavo- non volevo sottovalutare nulla. In anni in cui si parlava spesso, e forse troppo corrivamente, di morte dell'arte, di fine della storia, mi sembrava una grandissima qualità, per le sue possibili estensioni critiche o letterarie. Va aggiunto poi che conoscevo gli autori di persona. E sebbene non ci vedessimo che saltuariamente e casualmente (eravamo sparpagliati per il paese), mi sentivo legato a loro da un sentimento che andava oltre il rapporto di colleganza. Ricordo un incontro di una dozzina circa di noi - critici e scrittoriavvenuto nel 1986, a Vancouver, nel centro comunitario italiano, il particolare senso di amicizia e di solidarietà che animava le discussioni. Ci univa in quel momento un'ipotesi al contempo culturale e sociale, un progetto per intenderci sul quale le parole d'ordine che ci lanciavamo, termini come "etnicità", "multiculturalismo", poterano bastare e avanzare. In quel momento, credo, ognuno di noi avrebbe dato tutto perché quell'atmosfera non si disperdesse. E cosi, vuoi per spirito di bohème (si trattava dopotutto di un gruppo principalmente di scrittori), vuoi per diffidenza verso ogni burocrazia, vuoi perché nei dipartimenti la reazione verso le prime avvisaglie della letteratura italo-canadese era stata di scarso calore, quando verso la fine dell'incontro si diede vita alla Italian-Canadian Writers Association si decise di dotarla del minimo assoluto di struttura, di non tentare di affiliarci alle varie associazioni accademiche.

Dico questo non per narcisismo, per dare rilievo al mio tragitto intellettuale. Naggior rigore nell'individuare e formulare gli obiettivi del nostro gruppo avrebbe veramente nociuto? Si fece bene a prendere le distanze dalle istituzioni universitarie? Una maggior coesione avrebbe forse dato altri esiti in sede socio-culturale (insieme alle traversie dei singoli membri e alla scarsa capacità organizzativa, i conflitti interni ridussero di molto l'incidenza della Italian Canadian Writers Asssociation sulle cose letterarie o critiche di questo paese, che fu ed è pressoché zero). Certo, pure, una più pronunciata militanza istituzionale da parte di noi critici-docenti avrebbe permesso alla letteratura italo-canadese di circolare più facilmente nelle università. Nei dipartimenti di italiano, in cui molti di noi insegnavamo, un nostro attivismo avrebbe innescato subito, in anticipo sui tempi, una discussione critica che rimane quanto mai necessaria e che avrebbe portato nuova linfa all'italianistica canadese, avrebbe reso più vivace e più in sintonia con il clima dell'epoca (erano gli anni delle polemiche sul 
"canone", delle campus wars sul curricolo) il dibattito intellettuale nei comitati dipartimentali o nei simposi e nei congressi dedicati alla letteratura e alla cultura italiana. D'altro canto, qualsiasi nostro intervento dovera poggiare su basi che per noi erano includibili e irrefutabili, sulla nostra specifica percezione del nostro bic et munc, della nostra esperienza fuori del recinto universitario. E quel presente che noi cercavamo di chiarire a noi stessi era esattamente ciò che gravava sulle conversazioni con i colleghi, che non rientrava, non quadrava con l'immagine dell'italianistica promulgata e auspicata dai dipartimenti d'italiano.

Abbrevio: riflettere da italianisti sul ruolo della cultura dell'emigrazione costringe già in sede preliminare ad un radicale ripensamento del proprio lavoro e della propria biografia. Insegnare italiano nel Canada è operazione affatto diversa che insegnare in Italia. Il docente italiano può contare su studenti la cui identità culturale è già —o dovrebbe esser giàconsolidata da anni di scuola elementare, di ginnasio e di liceo. Lui stesso o lei stessa, quali che siano le sue interrogazioni intellettuali sul passato o sul destino futuro del suo paese, si trascina dietro quella sua identità meccanicamente, come se fosse un bagaglio naturale oppure un cimelio o un suppellettile da sempre in famiglia, senza dover auscultarla o dover tastarle il polso vita natural durante. Nel Canada, per un docente di italianistica di origine italiana la cultura italiana definisce insieme il suo raggio d'azione professionale (i soggetti su cui pubblicherà articoli e libri, i convegni a cui parteciperà) e il serbatoio di notizie, testi, istruzioni, rituali a cui attingere per coadiuvare un'identità privata che richiede cura e manutenzione continue. In questo l'iter dei docenti di italianistica di origine italiana non è dissimile da quello degli studenti, i quali molto spesso s'imbattono nella cultura dei loro genitori da adulti, intellettualmente, per la prima volta all'università. Né gli uni né gli altri si sottraggono ai dati della loro biografia: per loro l'italianistica è sempre una scelta doppia, una scelta anche sentimentale.

Ovverossia: l'emigrazione - e precisamente, con quelli di noi di origine italiana, per i suoi agganci con la nostra vita privata- apre il nostro piccolo recinto professionale al mondo e ai fatti del mondo. É la falla, la fenditura mediante cui la storia - questo ambito troppo a lungo negletto, forse, nei nostri programmi universitari o liceali-irrompe nel nostro tran tran quotidiano di studiosi. Ed ignorarla la si può solo a nostro rischio e pericolo, tirandoci fuori, cioè, da un discorso che ha un suo peso nel dibattito culturale nordamericano e quindi lasciando che siano altri ad enunciare, a rappresentare la nostra particolare esperienza.

Nel confrontarci con la storia e con quella conversazione in cui sono coinvolti i nostri colleghi nelle nostre università, ci troveremo subito a tu 
per tu con un noto e importante parallelo. Noi docenti siamo, oggi, nella situazione in cui furono esposti i nostri predecessori all'epoca di De Sanctis, ugualmente impegnati, per vocazione professionale, a formare, a "italianizzare" sé stessi tanto quanto i loro alunni. Non insisterò sul richiamo, benché gli equiroci e le suggestioni che da esso emanano darebbero già adito a paraggi culturali abbastanza significativi. Gli italianisti in paesi come il Canada non hanno, in quanto italianisti, una nazione di cui assillarsi. Hanno, al massimo, una identità che dovrebbe o potrebbe contribuire all'assemblaggio di connotati nazionali perpetuamente in fier. Si direbbe che sono votati nelle loro aule a diventare portavoce e promotori della comunità alla quale appartengono, nella misura in cui, volenti o nolenti, parlando di una materia che concerne essa comunità, la radicano nel paese ospitante. Ore venissero a cadere i dipartimenti d'italiano nelle università canadesi, verrebbe a mancare a tutti gli italo-canadesi un essenziale dispositivo di socializzazione. Volontaria o involontaria, l'adrocacy (per adoperare un vocabolo inglese intraducibile in italiano) degli italianisti ha dunque oggi una sua particolare risonanza intellettuale. Da un lato, come ogni insegnamento scolastico e il sistema educativo in generale, va assimilata all'ordine degli apparati di stato. Dall'altro, l'angolatura minoritaria dei suoi assunti mostra quanto insufficiente ed errata sarebbe una critica che, troppo ideologicamente rigida, concepisse quegli apparati in termini del tutto negativi. Ma il grumo di problemi verso cui finisce per orientarci una sia pur veloce riconsiderazione dell'italianistica canadese è di ancora più ampio respiro.

Nell'America del Nord i tagli, le riduzioni che hanno subito o che subiscono i dipartimenti di italiano non hanno a che fare sempre o principalmente con ta crisi budgetaria dell'università. Ci sono altre ragioni. In primo luogo, la caduta di prestigio della letteratura italiana. Nel modernismo degli anni venti o trenta, Cavalcanti e gli stilnovisti erano ancora fortemente citabili per autori come T.S. Eliot oppure Ezra Pound, che li consideravano figure inamovibili del canone europeo e mondiale. Oggi in concreto resistono Dante e, forse, il Rinascimento, sebbene in funzione museale, come generico supporto per gli studi del periodo elisabettiano inglese. E c'è chi riferendosi alla letteratura italiana parla apertamente di letteratura minore1. In secondo luogo, i dipartimenti di italiano risentono, come tutti i dipartimenti di lingue eccetto i dipartimenti di inglese e, nel Canada, i dipartimenti di francese, delle ripercussioni di un improvviso e drastico mutamento di rotta gnoseologico. L'arvento della teoria in tutti i rami del sapere accademico, l'ubiquità dei testi audiovisivi ha alterato in maniera drastica la nozione di disciplina, da identificare sempre più, e forse 
ormai esclusivamente con un medium o con delle normative metodo, logiche, con pratiche improntate ad una precettistica forte e di stampo scientistico o scienticizzante. Sono discipline i film studies e la storia dell'arte oppure la sociologia, la psicologia, le scienze politiche, la linguistica, non litalianistica, la germanistica, l'ispanistica, declassate ormai a campi di ricerca entro cui "applicare" ora tali ora talaltri protocolli di analisi (appunto i protocolli degli studi mediatici o quelle delle "vere" discipline). Tanto più che la divisione in categorie nazionali sembra poco giustificabile nell'epoca della globalizzazione, in un mondo segnato come nai prima da spostamenti demografici $\mathrm{e}$ in cui i prodotti culturali possono viaggiare alla velocità della luce, senza fermarsi davanti a nessuna frontiera. Di che sorprendersi allora se la materia dei corsi di italianistica riappare sotto altre forme in altri dipartimenti, se alle proteste per le chiusure dei dipartimenti di italiano le amministrazioni universitarie rispondono che lo studente a cui la cultura e la lingua italiane stanno a cuore potrà trovarle nelle discipline che hanno sempre ponderato e ponderano tutto ciò che è di natura storica o mass-culturale o glottologica, anche se l'italianistica non esiste più in quanto aggregato curricolare.

Contro quanto di sussicgoso e di mal digerito, sul piano intellettuale, nascondono gli smantellamenti dei dipartimenti di lingue straniere non mancano, per gli italianisti, postazioni di difesa rispettabili. Antico o moderno il medium dei suoi oggetti di studio - e nessuno in un mondo come il nostro può ignorare la consistenza materica di un prodotto culturaleuna disciplina la si istituisce e la si diffonde con le parole. Lo specialista non trasmette il suo sapere sul cinema o sui giochi video visivamente, emanando filmati. Comunica generalmente a voce o per iscritto. I precetti della disciplina li desume da articoli, libri, opere in lingua (in italiano, inglese, francese, tedesco, e via di seguito), ligi a tutte le costrizioni tropologiche o retoriche o stilistiche a cui obbedisce ogni discorso verbale. E gli strumenti disciplinari: è forse un inoppugnabile rantaggio averne, poter parlare di "società" o di "psiche" o di "cultura" in astratto, fingere che ce ne sia solo una di ciascuna. Però dopo come comportarci quando ci troviamo di fronte a questa o quella società, questa o quella cultura - le socictà, le culture al plurale, le società e le culture nella loro esistenza storica? In tale loro manifestazione sembrano piuttosto essere entità situate, che si dischiudono ad un sapere esso pure situato. Il sillogisma su cui poggiano le decisioni degli amministratori universitari si puo ribaltare. le discipline non esistono in astratto, interamente al di là della cronaca: c'è una tradizione francese in sociologia, così come c'è un'antropologia americana o una medicina cinese che si può comparare con altre tradizioni di altri 
luoghi e altri tempi. E poiché, in una suo certo qual modo, situato è il sapere, situati sono anche gli studiosi che a determinati saperi di determinate tradizioni devono il loro identikit professionale e intellettuale.

Tirare in ballo la globalizzazione e gli annessi sconvolgimenti avvicina al trambusto quotidiano, allo spirito dell'epoca in cui viviamo, ma non inficia queste conclusioni. Se può convenire ai politici, pronti a giustificare qualsiasi loro insufficienza con lo scaricabarile della mondialità dell'economia e della cosa sociale, una troppo disavveduta glorificazione delle magnifiche sorti e planetarie urta contro limiti di fondo. Come arverte Anthony Smith, lo studioso del nazionalismo, non possiamo avere memoria del mondo in quanto tale; abbiamo solo memorie particolari o particolarizzanti (23-24). Siamo, si potrebbe aggiungere, condannati all'identità dalla nostra stessa carenza cognitiva. Che l'informazione circoli a ritmi vertiginosi, non vuol dire che essa non sia soggetta a perdite o ad erosioni. Gli esseri umani i dati che ricevono li interpretano, li coniugano con altri elementi, li dimenticano, li interpretano, e li coniugano e li dimenticano perché così funziona il nostro dispositivo mentale e quella mente oggettiva che è la nostra cultura. Una diffusione uniforme, senza sbavature, senza sfasature, senza localismi non è ancora immaginabile, nonostante l'impeto omogeneizzante dei media elettronici e il percorso a senso unico (dal mondo del benessere al mondo della povertà, dal Nord al Sud) della globalizzazione informatica. Neanche sugli aspetti economici, che dovrebbero essere la più flagrante e incontestabile prova dell'avvenuta globalizzazione, l'accordo è totale. Lo strapotere delle multinazionali, ritengono alcuni specialisti, non annulla lo spazio di manovra dello stato; anzi il vecchio, stan$\mathrm{co}$, acciaccato stato può, nel nostro fine secolo, ripigliare fiato, dimostrarsi, curiosamente, se i cittadini lo rolessero, efficace baluardo di resistenza all'arbitrarietà selvaggia del mercato (Hirst e Thompson, 171-194). Alla stessa stregua, tutto lascia credere che lo stato e la nazione continueranno ad essere necessarie coordinate del sistema geo-politico, malgrado le notizie sul loro imminente decesso e malgrado l'indebolimento della loro sovranità e l'arrivo sulla scena di formazioni sovranazionali di impianto politico o economico (Unione Europea, Sud-Est asiatico, eccetera. Vedere Evans, Jones e Sterenson).

Ognuno di noi è preso in un insieme di aneliti e affiliazioni contrastanti, tra qualche speciale cosmopolitismo e qualche speciale localismo, e sarebbe errato credere di poter tagliare la testa al toro con una decisione che valga una volta per sempre, a favore di questa o quella singola opzione senza semplificare, senza togliersi dal gioco. La lezione della storiografia è che la storia si dipana e si studia sempre su scale diverse, che si intersecano ma hanno anche una loro autonomia, sia pur relativa e mai assoluta: si va 
dalla civilizzazione con la sua compagine internazionale, globalizzante (secondo lo storico Samuel Huntington le civiltà del mondo sono nove: l'occidentale, l'africana, la cinese, la latino-americana, quella indù, quella islamica, quella ortodossa, quella buddista e quella nipponica, $21 \mathrm{sgg.}$.), alla nazione, alla regione dentro la nazione, alla città?. Risultato: le frontiere, invece di scomparire si moltiplicano, direntano mobili, anch'esse di scala diversa. Per cui lo studio della differenza, delle stratificazioni socio-geoculturali conserva la sua attualità. Ponendo l'accento sul metodo, sui protocolli, si rischia di insegnare soltanto la propria cultura, di far passare per universali fattori che sono invece locali, di riorganizzare facoltà, dipartimenti, il sapere universitario secondo criteri di matrice eminentemente ideologica. É quello che accade spesso nel Canada. A nome dell'interdisciplinarietà, che può in se stessa arere sviluppi molto positivi, si sanciscono programmi di laurea, un sistema universitario in cui non c'è quasi più posto per nessuna altra lingua oltre l’inglese, si degrada ogni attività culturale improntata all'idea di traduzione (non è necessario conoscere altre lingue o altre culture se il nec plus ultra è conoscere altre discipline o l'andirivieni sovra-disciplinare). Sempre a nome dell'interdisciplinarietà e dell'erosione dei confini socio-politico-culturali si possono dissolvere o agglomerare in istituti di più vasta capienza le lingue straniere, e non i dipartimenti di inglese o di francese, che rimangono cattedrali intoccabili. Si risparmiano le sezioni di studi canadesi, come dovrebbe essere, per esigenze di politica sociale, invocando it ruolo della cultura nel promuorere l'unità nazionale, mentre si nega o si mortifica la rilevanza dei medesimi concetti, in ossequio alla regola della globalizzazione, quando si tratta di altri paesi e di altre culture.

Ora a tutto questo - a riesaminare il nostro coinvolgimento, a rimuginare le premesse geografiche della nostra professione per rivalorizzarne lo statuto di sapere in quanto sapere di altre culture e per meglio ricollocarla all'interno dell'assetto disciplinare - ci si arriva quasi ineluttabilmente. Alla lunga vi ci condurranno i giochi di potere che contraddistinguono la vita universitaria canadese (o nordamericana) e che hanno anche tinte culturali, se non propriamente etniche. Ma, va da sè, nel risituarsi rispetto al Canada o all'America del Nord, gli italianisti canadesi si risituano simultaneamente rispetto all'Italia. I due processi si presuppongono a vicenda.

Molti tra quelli di noi che sono nati in Italia, sono qui nel Canada, come lo sono io, da alcuni decenni. Pendolari o no (lo so, ritorniamo spesso in Italia), abbiamo stabilito in questa sponda dell'Atlantico la nostra residenza. Anche in questo, nonostante i divari di classe, che ci sono (molti di noi però provengono da famiglic di operai o di contadini), la nostra fedina la condividiamo con i nostri connazionali o con i nostri studenti di 
origi-ne italiana che vivono fuori del campus. Nemmeno, noi possiamo amman-tarci di eccezionalità romantiche. Non siamo nè esuli, nè profughi, nè nomadi in moto perpetuo ma immigrati.

A conferirci il nostro tasso di dignità storica è la particolare separatezza che incarniamo. Noi siamo tra coloro per i quali l'Italia è un'origine, una realtà distaccata, a cui apparteniamo con (e non cancellando) la nostra assenza. Che è un'assenza defilata, "bassa", poco epica - come può esserlo quella di chi riconosce i propri monumenti in Ellis Island o nel molo del porto di Halifax, nelle Little Italy metropolitane- e tuttavia modernissima: l'assenza delle folle che hanno popolato, costruito e rivivificato le città più à la page (che cosa erano New York o Montreal o Toronto prima dell'emigrazione?). Siamo tra i cliché del secolo, tra la galleria di tipi, le dramatis personae di cui si compone il teatro quotidiano dell'epoca, come lo sono il magnate dell'industria o dell'alta finanza e il cantante o la cantante rock, lo scienziato e il nobile decaduto, l'operaio e la casalinga, il segretario di partito e l'annunciatore televisivo, la stella cinematografica e il papa e il campione sportivo, e il resto della lista compilata, aggiornata e chiosata a varie riprese da sociologi, antropologi, scrittori, opinionisti e caricaturisti maggiori e minori. Intellettualmente, nella nostra specifica temperie culturale, il primo, impellente compito di noi docenti d'italiano fuori d'Italia che siamo di origine italiana è come recuperare a vantaggio della disciplina il nostro bifocalismo.

L'emigrazione, in che modo, dunque, cambia la materia che insegniamo? Soffermiamoci un attimo sull'effetto concreto più vistoso. Non appena si colloca l'emigrante (o il docente emigrante) dentro l'orizzonte dell'italianistica, questo orizzonte si espande e quantitativamente e qualitativamente. L'Italia del nostro fine secolo possiede già nel suo interno una geografia noetica, virtuale, lontananze che alterano la sua ingredienza geoculturale. Sono quelle che evocano i visi, le parole, le usanze e i costumi degli immigrati, dei nuovi Italiani o degli Italiani delle generazioni a venire. Con l'emigrazione si procede nella direzione opposta: all'Italia si giustappongono gli spazi abitati dalle popolazioni italiane sparse per il mondo. Entra nel perimetro dell'italianistica ogni zona in cui rimangono tracce di un qualche duraturo passaggio di gente italiana. Più che l'Italia, ormai l'oggetto di studio dell'italianistica sarà la cultura dell'italianità, passata, presente e futura. Cioè, la cultura italiana intesa nella veste, nell' ampiezza mondializzante e mondializzata che nel nostro tempo le compete.

Se dividiamo, come stiamo facendo, e come faremo, questo spazio e puntiamo per convenienza euristica il nostro cannocchiale sull'Italia fuori d'Italia e sul suo contributo, a scapito della visione totale (dell'italianità 
demografica, che comprende l'Italia come è stata finora, l'I talia degli immigrati e i luoghi da cui essi provengono), non ì importante distinguere subito tra il rimaneggiamento che arviene per autorappresentazione e quello che arviene per rappresentazione, per autobiografia o per biografia, per mano propria o per mano altrui. Litalianistica la si puo professare in varie guise, e la pluralità delle ottiche sta li, se la si giudica positivamente, ad indicare la ricchezza, il potenziale prismatico, polifonico della disciplina. Nel Canada un dipartimento ha tutto da guadagnare a mescolarsi, ad includere, quando può, docenti italiani, docenti italo-canadesi e docenti di altra estrazione culturale ${ }^{3}$.

Una veloce ricognizione dell'italianità fuori d'Italia ci porterebbe dall'arcipelago egeo, all'Eritrea, alla Somalia, all'Etiopia, alla Libia, dalla Svizzera alla Germania, alla Francia al Belgio alla Gran Bretagna, dall' Imerica del Sud, all'America del Nord, all'Australia e al Canada. A classificare secondo i connotati più sommari e generici, affiorerebbe quasi immediatamente in questa nostra panoramica dell'Italia fuori d'Italia una suddivisione tra topografie coloniali e topografie migratorie di grande complessità (nelle colonie la cultura italiana è la Legge, si presenta principalmente in veste coercitiva, sotto forma di editti che hanno l'obiettivo di favorire la gestione politica del territorio; le Little Italy sono luoghi informali, nonlegislativi, tenuti insieme da motivazioni puramente culturali, non hanno nessun peso politico, né cullano secondi fini politici). E si pensi ai caveat e ai distinguo a cui darebbero adito ulteriori graduatorie. L'occupazione-colonizzazione dell'Egeo ha un retroscena pre-moderno; quella della Somalia postumi recenti (il paese africano è stato protettorato italiano nel dopoguerra). Essere più vicini o meno vicini dall'Italia, dentro o fuori l'Europa ha una sua pertinenza per gli emigranti. In paesi come la Francia o la Germania, con una identità nazionale forte, che assimila i nuovi arrivati o li relega nella categoria di gastarbeiter, "lavoratori ospiti", i quartieri italiani, se ci sono, non hanno la risibilita, la specificazione simbolica che hanno aruto e hanno oltreoceano. In Europa, con la comparsa dell'Ĺnione Europea la nozione di emigrato/immigrato assume varie sfumature, tende ad essere associata sempre più con gli extra-comunitari; i cittadini con passaporto europeo che si trasferiscono al di là dei confini nazionali godono di uno status giuridico interamente diverso da quello che avrebbero negli Stati Uniti o nel Canada o in Australia. Falsarighe sempre locali seguono, nelle settler societies, nei paesi che hanno conosciuto colonizzazione ed emigrazione, i contatti tra gli emigranti italiani e i gruppi che li hanno preceduti. Il continente latino-americano, ispanizzante e cattolico, non è il mondo anglosassone e protestante del Commonwealth: benchè negli Stati 
Uniti si parli spesso oggigiorno di "Euro-Americani", tra il gruppo dominante per censo e potere, i WASP, e gli Italo-Americani non è mai esistita quella confidenza che si riscontra tra i Latino-Americani di origine spagnola o portoghese e quelli di origine italiana, accomunati da affinità linguistiche, oltre che religiose.

Ma il fulcro, il motore di questa geografia culturale, di questo spazio discontinuo, intervallato, è il rapporto con l'Italia. Lo è ovviamente per i paesaggi del post-colonialismo italiano (non sono stati i soldati di Canicattì a pacificare la Cirenaica o a marciare in Addis Abeba), e lo è per il fuori d'Italia dell'emigrazione, dove pure i legami con il presente e il passato italiano sono più sfaccettati. Confermo le mie riserve circa l'utilità storica, negli anni dell'economia di mercato e della globalizzazione, di inveire contro lo stato-nazione o di elicitare dagli spostamenti demografici dell'ultimo secolo facili profezie su un futuro interamente post-nazionale, entrambe pratiche in cui indulge con molto diletto molta critica sociale o letteraria e che appiattiscono, semplificandolo, lo stato delle cose. Chi è fuori d'Italia e dissolvesse nella sua mente l'Italia in quanto territorio politico con una sua cultura e una sua storia si priverebbe di un riferimento decisivo, e non tarderebbe a soffrirne sul piano sociale e/o politico. A seconda delle circostanze si possono appagare le proprie ansie identitarie restringendo lo spazio, richiamandosi al quartiere o al paese o alla regione. Succede cosi ogni volta che ci si vuole differenziare da altri connazionali o altri corregionali o persone della nostra stessa città. Ripiegare su simboli collettivi di scala inferiore a quella nazionale, e quindi di non facile collocazione, è meno conveniente in altre circostanze. Le quali sono ancora, per lo più, a sottofondo inter-nazionale. Sbagliarci di ambito ci lascerebbe alla mercé di persone e gruppi che hanno meno tentennamenti, oppure mortificherebbe il nostro ruolo. Se è vero che l'identità è multipla e che i suoi contenuti sono contingenti, prorvisori, suscitati dall'incontro o dallo scontro con i nostri simili, nel processo di produzione dell'identità agisce anche un principio irenico. Identificandoci, noi contribuiamo al formarsi dell'identità altrui, e vice versa. Nella sfera pubblica non aiuta nessuno, né noi né in nostri interlocutori, dire "Sono friulano" oppure "Sono pescarese", oppure "Sono di Porta Romana" quando i criteri a cui magari ricorre chi ci ascolta si riallacciano a luoghi (e agli eventi, ai costumi, alla cultura ad essi associati) di maggiore ampiezza e di maggiore riconoscibilità.

C'è una ragione ancora più incalzante, non del tutto disgiunta dalla precedente, perché nel rimuginare i rapporti tra il fuori d'Italia e l'Italia si debba privilegiare la nazione. È che la nazione, essa, orienta già di per sé, tramite i suoi apparati, i rapporti in tale direzione. Si sa, l'intellighenzia uffi- 
ciale italiana ha abbandonato il tema del'emigrazione all'estro e alla buona volontà dei singoli studiosit. Come per il colonialismo, su questo argomento, i manuali scolastici, le storie della letteratura o della cultura continuano a tacere. Sembra che l'esistenza di sessanta milioni di Italiani fuori d'Italia, un numero ormai superiore al totale della popolazione della penisola, sia senza significato, non meriti di far parte dell'equazione (la più recente rassegna dell'identità italiana, quella di Ennesto Galli della Loggia, riesce ad evitare perfino l'accenno di prammatica). Più positiva, una rolta tanto, la politica culturale dello stato. I lettorati, le borse di studio, gli istituti di cultura, gli accordi sugli scambi culturali, sulla doppia cittadinanza, le borse per gli studenti universitari sono iniziative concrete che influiscono sulla formazione del futuro ceto dirigente delle comunità migratorie e valgono più di ogni retorica sui Comites e sul diritto al voto. Ma costituiscono, alla fin fine, il minimo indispensabile. Deliberata o no, la strategia dello stato italiano mira a salvaguardare un patrimonio: è la strategia del tutore. In questo schema, tra la nazione e il fuori d'Italia non sussistono fratture. Agli emigranti è affidata una delega - quella di rappresentare l'Italia - che l'Italia provede a tenere ben aggiornata c sulla cui sostanza essi non hanno voce in capitolo. Un atteggiamento, questo, che per un'italianistica aggiornata non può per niente essere "naturale" o scontato. L'irritazione dei dignitari italiani nel constatare che spesso gli emigranti custodiscono in sé un'immagine dell'Italia molto anacronistica è il rovescio dell'irritazione degli emigranti nel vedersi sempre ritratti sul predellino del treno con la valigia di cartone, nella solita istantanea in bianco e nero, per sempre immutati e immuteroli. Ambedue reazioni vanno vagliate, interpretate alla luce della storia. Che è una storia in cui l'Italia-nazione e l'italianità si sorrappongono ma non coincidono, in cui è possibile concepire un fuori d'Italia che ammette i suoi rincoli con l'Italia ed è costantemente in fuga da essa, al punto, magari, di parlare un'altra lingua. L'na storia in cui c'è posto per il dialogo, in cui il fuori d'Italia c̀ ormai portatore di un supplemento di cultura, di un accumulo di vicissitudini da cui l'Italia-nazione può trarre forse giovamento, forse tanto quanto il fuori d'Italia ne trae dai contatti con l'Italia.

Non proverò ad anticipare per filo e per segno i futuri programmi di questa italianistica dell'italianità e delle fratture e delle dialettiche che la contraddistinguono. Sul piano istituzionale sarebbe indubbiamente un'italianistica con un'organizzazione curricolare molto diversa, in reciproco accavallamento con quella di vari altri dipartimenti. Corsi o seminari sulla presenza italiana in $\lambda$ frica o nell'liuropa settentrionale o nell'America 
Latina o in Australia, analisi comparative delle varie comunità e del loro diverso legame con l'Italia allargherebbero il raggio pedagogico dell'italianistica, permettendo iniziative comuni con altri settori dell'università. Non sono solo i dipartimenti di inglese o di francese o di spagnolo che possono vantare una materia "internazionale"o "sovranazionale". Se il percorso istituzionale di questa italianistica si profila non meno lungo ed incerto, ’̀ l'unico percorso accettabile, e le sue potenzialità didattico-curricolari andrebbero almeno ventilate o illustrate alle autorità competenti (ottunderebbe le loro prevenzioni sulla cultura italiana, sulla sua scarsa "esemplarità" storica). Sul piano puramente intellettuale invece l'analisi del rapporto Italia — fuori d'Italia offre già casistiche estremamente ricche: ognuno vi troverà pane per i propri denti, potrà soddisfare la propria voracità, accaparrarsi i tracciati in più diretta attinenza con la propria arrenturosità intellettuale. Mi limiterò, sulla scia degli spunti offerti dalla cultura italocanadese, ad elencare alcuni dei crocicchi meno improbabili, alcuni dei topoi verso cui sembrano dirigersi i sentieri più frequentati dalla critica.

Da quando esiste l'italianistica, è stato il suo mandato istituzionale di accudire - e accudire per poterla trasmettere - a una tradizione letteraria millenaria, che da Francesco di Assisi giunge fino a Calvino, Baricco, Tabucchi, Dacia Maraini, Dario Fo e agli scrittori e alle scrittrici viventi che leggiamo e assegniamo ai nostri studenti. Passare dall'Italia all'italianità obbliga noi italianisti a ricalibrare lo spazio-tempo, i cronotopi della nostra materia, a ritoccare per la prima volta da un secolo in qua la superficie e la qualità del territorio a noi demandato, e di prendere atto dell'impatto che una ristrutturazione dello spazio avrebbe sulla componente temporale, quale è stata finora tratteggiata. Riveduta dal fuori d'Italia italianizzante, la tradizione che amministra la nostra disciplina si allunga. Non combacia esclusivamente con lo scopo (contribuire all'unificazione reale del paese) a cui è stata adibita nel clima che l'ha "inventata". Condicio sine qua non, nocciolo irrinunciabile anche temporalmente, la nazione non è il tutto. Il corpus dell'italianistica si erge sul grande tronco della classicità latina. Virgilio, Seneca, Plauto, Orazio, Ovidio sono suoi antenati. Il suo è il più poliedrico e tortuoso e ininterrotto itinerario della cultura occidentale, un itinerario che abbraccia molteplici esperienze politiche, sociali, linguistiche, culturali sorra- e intra-nazionali, che può illuminare le questioni da cui è travagliato il nuovo millennio e simultaneamente esserne illuminato. L'Italia medievale, insieme latina, bizantina, normanna, longobarda e araba, ci apparirà forse di sembianze abbordabili e improrvisamente fascinose alla luce del dibattito sul pluralismo etnico nella nostra società ma 
storicizza tale pluralismo e tale dibattito. Pur forse sotto mentite spoglie, ì sempre l'incontro con l'Altro, e spesso con l'Altro nella variante islamica che sembra volersi foggiare oggigiorno la cultura europea contemporanea, il tema di maggior spicco dell'epopea nostrana, da Virgilio a Boiarclo ad Ariosto a Tasso. Per superficie l'impero romano, il Sacro Romano Impero, il regno terrestre che si prefigurava la Chiesa dei papi e dei missionari controriformistici forse non reggono il paragone con il Commonnealth britannico o la Francophonie: erano però strutture, sistemi globali o globalizzanti, se proporzionati ai mappamondi dell'epoca. E a provocare la rentilata rivalsa del municipalismo saranno marchingegni indubbiamente norecenteschi —l'economia transnazionale e trasversale, l'irreversibile porosità ed erosione dei confini politici, il vertiginoso viavai dell'informazione elettronica; ciò non toglie che, quando le predizioni si verificheranno - se si verificheranno- i precordi che verranno in mente saranno le città-stato rinascimentali e i comuni tardo-medievali.

Nella letteratura italo-canadese la nuova italianistica —l'italianistica pronta a caldeggiare l'intero grafico della temporalità allargata a cui sovrintende e a coniugarlo con lo spazio oltre l'Italia che aprono i sommorimenti demografici moderni- scorgerà subito alcuni addentellati con l'Italia da sfruttare criticamente. Ci sono, come è da aspettarsi, affinità elettive innanzitutto con il lungo plurisecolare filone del fuoriuscitismo letterario. Penso non solo ai grandissimi, a Dante o a Petrarca o al Leopardi che si "fingeva" l'al di là della siepe, ma anche ad autori come Foscolo o Parese o Meneghello, oppure, ancora, al largo ventaglio di scrittori meridionali che, da Verga a Pirandello, da Alvaro a Silone a Vittorini a Quasimodo, hanno conosciuto l'esilio, la separatezza, l'emigrazione e li hanno descritti con acume e sensibilità, per i quali l'esilio, la separatezza o l'emigrazione sono stati esperienze quasi istituzionali, erano la regola del mestiere, risto che nell'Italia dell'Otto-Novecento si esercitava —e si è esercitatosoltanto previo soggiorno nel continente o al Centro-Nord. Per quanto la critica italiana abbia rimosso o non abbia quasi mai preso sul serio quelli di loro che hanno conosciuto il Norecento (emblematico qui l'affaire Silone), essi sono, ora che su termini come "dislocazione", "diaspora", "memoria", "viaggio" è imperniato il vocabolario di base di ogni discorso intellettuale, letterario e no, gli scrittori più moderni dei moderni, per adoperare la bella espressione di Pasolini, un altro autore a cui i problemi, le ricissitudini che questo rocabolario rispecchia non erano ignoti. Ii sono essi gli scrittori, oggi, che toccano più da vicino chi si occupa di letteratura in paesi come il Canada, o gli Stati Uniti o I'Australia. Non per nulla, tra l'altro, è nelle opere di alcuni di loro che viene delineato nella sua rersione letteraria più 
matura il mito dell'America, alle cui lusinghe obbedirono massicciamente gli emigranti italiani e il cui fascino sembra già lasciar presagire il tardo novecento e il ventunesimo secolo, la centralità mondiale, la possanza neocolonialistica dell'economia o della cultura americana.

Na questi agganci si coniugano, criticamente, per noi che leggiamo da prospettive non puramente o non interamente europee, con due altre simmetrie. La prima rimanda al Duecento/Trecento, a quel crogiuolo prenazionale di lingue (latino veicolare, provenzale, dialetti vernacolari), gerarchie geo-politiche (impero, chiesa, comuni, regimi vari) e culture in cui s'inquadrano la poesia siciliana e la poesia toscana, i testi che convenzionalmente inaugurano la tradizione italiana in italiano. La seconda rimanda all'Ottocento, ad autori come Foscolo, Nanzoni, Leopardi, Verga, alle dispute sul romanticismo, sull'unificazione politica e culturale, sul concetto di letteratura nazionale o di letteratura regionale in auge immediatamente prima, durante e immediatamente dopo il periodo risorgimentale.

Non si peccherebbe, credo, di eccessiva sudditanza verso lo spirito del tempo se si riunissero queste analogie sotto l'egida dell'emergenza, se si scorgesse nel loro intrecciarsi un incitazione a riflettere sul momento embrionale o, meglio, sui momenti embrionali della letteratura. A suggerirci questo ulteriore assestamento non sono soltanto i reperti sui testi o sugli autori italo-canadesi, per i quali abbiamo date di pubblicazione recenti (intorno alla metà degli anni settanta) e carriere ancora in progress, che possiamo seguire con relativa facilità (sono tutti scrittori viventi). Emergere nel romanzo, nella poesia, nel teatro attesta di una complicità che mette sempre i materiali di base della letteratura in stretto rapporto con la storia del gruppo.

Le domande preliminari con cui si scontra chi si accosta alla letteratura italo-canadese insieme evocano e allargano quella questione della lingua sorta con i poeti e con i narratori del Duecento/Trecento e riacutizzatasi poi nell'Ottocento quando romanzieri e i drammaturghi si trovarono a dover decidere come far parlare i personaggi degli strati sociali o delle terre ancora incognite dell'Italia ai quali essi cercavano per la prima volta di dar voce. Perché la stragrande maggioranza degli scrittori italo-canadesi scrive in inglese o in francese, e non in italiano? Se l'impasto di lingue non è nemmeno ipotizzabile nel Canada o negli Stati L'niti, cosa dedurre a proposito della letteratura, della letterarietà di questi paesi? Che significato attribuire ai titoli in italiano che serpeggiano (venti su un totale di ottanta poesie) in The Tough Romance, seminale raccolta di Pier Giorgio Di Cicco? Qual'è il ruolo dell'italiano nell'ordinamento linguistico mondiale all'inizio del terzo millennio: Come riformulerà la questione della lingua l'italianistica che dorrà cimentarsi, oltre che con l'Italia fuori d'Italia, con l'arrento dell'in- 
glese, quando esso, cone sembra accadra, diventerà la lingua ufficiale dell'Unione Europea?

Ad alcune di queste domande si puo rispondere senza troppe tergiversazioni. Nel Canada in italiano scrivono gli emigranti arrivati in età adulta. Sono per lo più scrittori che si rivolgono a lettori all'interno della comunita, e le cui opere circolano in un'ambiente molto ristretto: giornali a piccola tiratura, editoria comunitaria. Scrive in inglese o in francese la generazione dei figli, siano essi renuti nel Canada nell'infanzia (una grossa fetta degli autori) o canadesi di nascita. Mentre i testi in italiano svolgono, per i loro fruitori, mansioni principalmente fàtiche, cioc che rafforzano il contatto ${ }^{5}$, permettendo ai lettori di trasformare la comunità in una comunità immaginaria, $i$ testi in inglese o in francese si prefiggono come scopo quello di iscrivere la comunità nell'ecumene canadese.

È per questo che questi ultimi hanno più da spartire con la letteratura italiana - e con la letteratura duecentesca, trecentesca e ottocentesca in particolare, ripeto- di quanto non ne abbiano i testi in italiano. Tramite la scrittura si nominano, si registrano, si sedimentano ricende, eventi e individui, nell'archivio collettivo di una società. Si rappresenta, si proclama, si certifica una presenza prima non detta e che altrimenti sarebbe scomparsa nel tempo. In un certo qual modo si portano anche notizie, o, se si vuole - per ricordare un genere apparso con il nascere della letteratura in vernacolo italiano- "novelle". D'altronde nel Duecento o nel Trecento la letteratura sconfinava volentieri nella "cronica" o nella "istoria", e le "croniche" o le "istorie" nella letteratura. Per l'Ottocento valga su tutti, in Italia, l'esempio di Manzoni, che impiegò il suo talento argomentativo per mostrare che ogni romanzo, ogni finzione letteraria, era storica.

Va osservato che, su questo piano dell'uso della lingua, della funzione linguistica da privilegiare, né la condizione degli scrittori italo-canadesi che si servono dell'italiano, né la condizione di quelli che si servono dell'inglese o del francese è priva di pathos critico. Indirizzando i propri testi a lettori in Italia, un'alternativa con una sua attraenza, si rinunccrebbe al movente più giustificabile, a far riverberare i propri testi nella comunità italocanadese, e quindi a farorire il contatto. Per poter trasformare la scelta dell'italiano in un progetto di resistenza culturale occorterebbe, a chi avesse la sufficiente consapevolezza storica o letteraria, una tolleranza quasi monacale della solitudine (o forse un altrettanto ipertrofico estetismo: fuori d'Italia, come lingua letteraria, l'italiano avrebbe un'estraneità perfino più accentuata della diversità che ha avuto e ha il dialetto della poesia dei vari Pasolini, I.oi, Pierro). Sarebbe comunque una scelta non attuabile. Nel Canada la popolazione italofona è sempre più esigua: si compone soprattutto di genitori, persone poco istruite, con una padronanza molto pre- 
caria dell'italiano, oppure, ora che il flusso migratorio si è arrestato, di professionisti di passaggio, di persone che non hanno nessuna intenzione di fermarsi, di trapiantarsi. Nancano, semplicemente, la massa demografica necessaria per creare una vera tradizione letteraria in italiano, e le abitudini di lettura necessarie per poter effettuare deviazioni o scarti.

Al tradimento culturale sono votati invece per definizione gli scrittori che scrivono in inglese o in francese e traducono le Little Italy per i lettori canadesi. Le loro opere innestano spesso - molto spesso - le vicende dei figli o delle figlie su quelle dei padri o delle madri. Prima generazione italocanadese ad avere accesso alle scuole superiori, ai corsi universitari, è questo il loro regalo alla generazione precedente, che non avrebbe mai potuto stilare la propria biografia. L'omaggio non viene gratis. La generazione dei vecchi è stata indubbiamente raccontata dalla letteratura italocanadese anglofona e francofona ma non è tra i suoi destinatari. Le parole stesse che la narrano -inglesi o francesi- l' avrebbero esclusa, non fosse pure essa già penalizzata dalla sua proverbiale renitenza alla lettura. Nelle Little Italy sono -oltre agli "altri” — gli acculturati, i figli e le figlie, non i genitori, il pubblico del testo italo-canadese anglofono o francofono. Il prezzo da pagare - in contanti, e subito - per il diritto a mettere nero su bianco, ad infiltrare il canone, il catasto delle rappresentazioni è l'incomunicabilità intergenerazionale, la frattura tra casa e scuola, tra presente e passato. Poiché non sono riusciti ad appropriarsi dell'inglese, ad agire su di esso (non esiste l'equivalente italo-canadese dell'ebonics degli statunitensi di origine africana, o dell' Indian English o del Caribbean Enghish, un italoinglese codificato, parlato dalla comunità), gli emigranti italiani nel Canada sono un gruppo senza lingua, o, forse, tra le lingue. Nei testi l'italiano non può intervenire che come un'assenza, una perdita, un residuo. È ciò che ci indicano i titoli delle poesie di The Tough Romance e gli spezzoni di frasi in italiano che fanno capolino di tanto in tanto nei romanzi, nel teatro, in altre poesie italo-canadesi. Ed è, questo insistito intrudere di frammenti di lingue straniere nella norma linguistica di un paese, uno dei fenomeni su cui un' aggiornata questione della lingua dovrà soffermarsi (si possono considerare suo riswolto tipologico le molte parole inglesi che punteggiano i giornali italiani, un'intrusione da una posizione di dominio).

Tematicamente si offrono come spia più flagrante delle antinomie degli scrittori italo-canadesi anglofoni o francofoni i molti ritratti di famiglia che essi ci hanno tramandato. Per noi che abbiamo avuto come viatico mentale Freud e la psicanalisi non è facile accostarci al particolare romanzo famigliare che ci allestisce la letteratura italo-canadese senza ricondurlo al solito crinale edipico su cui si è attardata la tradizione maggioritaria del Norecento. Ma già la prima tappa dell'intreccio - la lotta con 
i genitori, il padre principalmente- yui è scandita da cadenze più sociali che private, da una incomunicabilità che deriva patentemente dalla condizione storica dei personaggi. "N/ contrario di sua moglie", dice di un genitore una poesia di Mary di Michele, "lui può parlare ai suoi bambini/ nella lingua in cui sognano, ma la tiene, questa lingua,/ nelle sue tasche come la chiave mal forgiata di una casa estiva" (132). In Linder The Bridge, secondo volume di una tetralogia di Frank Paci, l'incapacità dei genitori ad imparare l'inglese convince il protagonista Markie Trecroci ad isolarsi in un suo esilio adolescenziale, a compensare rifugiandosi in romanzi e fumetti, nel mondo di parole che gli promette la lingua del paese in cui è nato.

Molla di questa strategia dell' "allontanamento" (la locuzione è di George Amabile, 40), della tendenza dei personaggi a vivere i passaggi psico-sociali secondo lo schema del viaggio, è un impulso cruciale. Il tema di più cospicua valenza nella trama di cui si nutrono la narrativa, la poesia e il teatro italo-canadese anglofono o francofono sostituisce - di nuovo, significativamente per le sue risonanze letterarie - il ricordo traumatizzante, ricorrente della scena originaria psicanalitica con le promesse dei miti della fondazione. I giovani italo-canadesi, si direbbe, sentono prima di tutto il bisogno —o forse è un dovere — di confrontarsi da sé con la realtà, di ricalcare in qualche maniera i primi gesti e i primi eventi da cui nascono società e nazioni. In Toni, romanzo di Fiorella De Luca Calce, alcuni ragazzi e ragazze si riuniscono in una recchia casa di Montreal: insieme ricostruiscono una parrenza di gruppo e di ordine sociale sganciato dalla famiglia. In altri romanzi l'ambientazione meno urbana renderà ancora più palesi gli intenti dei personaggi. Su invito di un ex-compagno di scuola italo-canadese, il protagonista del romanzo di Paci già citato si ritroverà nella fitta boscaglia dell'entroterra ontariano per la festa del ringraziamento. Ia onorerà, con la comune amica di ceppo irlandese che lo accompagna, in un rituale indigeno officiato dal loro coetaneo. La sortita nel rasto outdoor canadese, di tuttora incontaminata edenicità, "come l'ha fatto Dio"(285), gli si offre come pretesto per riscoprire il paese, per ripetere il momento del primo contatto dei pionieri europei, ma da emigrante, capovolgendone il senso, con l'uomo bianco che adesso mima l'indiano, si nativizza volontariamente (l'accezione primaria del rito la annuncia, nel racconto, un incontro con una guida ojibway). Arendola intrapresa, lui, Narkie Trecroci, potrà ormai considerarsi "più canades $|\mathrm{e}|$ di coloro che l[o] arevano precedut[o]" (296).

Vale la pena sottolineare che lo stratagemma è doppio, forse triplo. Il riaggio di partenza, lo staccarsi dai genitori non esaurisce l'epica ordinaria, non-eroica, non-aristocratica di cui la letteratura italo-canadese si incarica di iscrivere le peripezie. Nell'instaurare in uno spazio primordiale la 
società dei giovani, della fratellanza e della sorellanza, sotto l'egida di Antigone, invece che di Laio o Giocasta o Creonte -incarnazioni di un'autorità sovrana, indiscutibile — si rigetta il modello colonialista, un modello che, in ultima analisi, esclude anche i genitori storici dei personaggi (gli emigranti, pur avendo spalleggiato l'espansionismo europeo, vi hanno partecipato dal basso: non hanno portato con sé gli eserciti, e non sono stati loro ad imporre la loro legge sugli indigeni). La migrazione dei figli e delle figlie istruite dalla Little Italy è uno dei segmenti di un viaggio zigzagante, circolare. Prevede il rivolgersi indietro, il ritorno simbolico, le virate, non di rado conclusive, verso la famiglia. In Under The Bridge, l'estatica comunanza con il territorio in cui culmina il ringraziamento ojibway ispira all'io narrante un ringraziamento cristiano eseguito con sostanze il vino del padre, il pane della madre - che lui a casa aveva sempre rifiutato e che nella foresta lo riconcilia con il passato. Sicchè, canadesizzandosi, il protagonista di Paci (e della trama che, intera o a frammenti, sottosta alla letteratura italo-canadese), completa e dilata oltre il traguardo iniziale l'impeto alla metamorfosi, il sogno che avera motivato l'esodo dei genitori e che i genitori non erano riusciti a realizzare.

Come sempre, le singole opere moduleranno il loro materiale secondo il loro apposito telos. Accentueranno ora la lotta-allontamento ora il ritorno-identificazione oppure un incrocio dei due. Criticamente domandano forse, oggi, più attenta empatia —e proprio perché possono sfociare in letteratura molto convenzionale - i testi riconciliativi, imperniati sui genitori. I campioni più maturi e più sicuri di questo sottogenere ospitano una narrazione o una meditazione che ribadisce non solo i tratti epici dell'intreccio migratorio in generale ma anche la specificità italo-canadese. Inserito nella geografia in cui viene ad inquadrarsi il racconto o che $\dot{e}$ implicito nella coscienza dell'io poetico, il Canada appare nella sua propria veste: è una delle province di quella utopia concreta, storica, localizzabile, che è stata l'America per il proletariato italiano. Ha ragione l'italo-statunitense Robert Viscusi quando, nel suo straordinario, meta-narrativo, fittizio resoconto dell'emigrazione intitolato Astoria, permette alla roce narrante di sostenere che gli emigranti hanno compiuto l'unica vera rivoluzione sociale dell'Italia moderna (294). Forse perché una più alta percentuale degli emigranti italiani è giunta nel Canada durante gli anni cinquanta e sessanta e quindi conserva una memoria diretta dell'Italia, la letteratura italo-canadese corregge l'ottimismo made in USA, dilungandosi con più deliberazione sull'amaro codicillo che si annida nel seno di ogni rivoluzione. Non per tutti i testi il riconoscimento dei genitori è gesto euforico. Lo impedisce spesso il ritorno rinterzato, maggiorato, simbolico, contraddittorio a cui sono votati i protagonisti dei romanzi o le ruminanti 
prime persone delle poesie, la loro identificazione con il ricordo dell'Italia che hanno il padre e la madre. Questo secondo ritorne, pendant narrativo del secondo "allontanamento" dei figli e delle figlie, dà la stura al lato distopico, esistenziale, ontologico dell'emigrazione. Nelle poesic di Pier Giorgio Di Cicco la memoria dell'Italia, si fa piú struggente é piú inderogabile via via che l'American drectm esplode, e la "decorosa felicità" (107) insita al mito dell' Imerica si dimostra chimerico miraggio.

Viene dunque riscattato quel tono che a volte pervade il tema dell'emigrazione nelle sue versioni popolareggianti (dalla canzonetta al cinema) e che ha pesato e pesa tuttora negativamente nel calcolo del valore estetico della letteratura dell'italianità fuori d'Italia. Contro una tradizione che rede nella nostalgia nient'altro che l'occasione di un'impudica lacrimosità, una specie di degrado della sensibilità, qui l'appello al passato si carica di assunti fortemente critici. Tic elementare quanto si vuole, quando all'utopia accertabile che è stata l'America si oppone un contro-mito altrettanto concreto, un passato ancora vivibile, si rivela un disagio, si adombrano le prime linee di una contestazione. La memoria "è un film troppo sentimentale per commiserare", dice Di Cicco, ma è "l'unica cosa che abbiamo per sentirci umani" (107). Se partecipare alla vicenda del proprio padre o della propria madre culmina nell'accettazione di quella comune umanità a cui si appellano anche i personaggi di Paci quando riaccolgono i genitori nella loro vicenda di figli canadesi, l'adesione contiene adesso una sua quota di disincanto, matura insieme ad una nuova, più realistica coscienza della propria biografia e dell'emigrazione nel suo spessore storico. L'America, spiegherà Di Cicco in un'altra poesia, è come una donna di cui non si può fare a meno. Chi l'ha conosciuta non si accontenta di una "moglie rispettabile". Eppure non è, questa donna, che "una buona puttana", "niente di cui innamorarsi"(109). In certa letteratura italocanadese, l'emigrante di seconda generazione si dibatte tra il mito e la storia. Il suo destino è il tongh romance che per Di Cicco ricapitola l'esperienza italiana dell'America, lo stoicismo epocale di chi, sebbene abbia beneficiato del salto economico assicuratogli dal nuovo paese, ha visto fallire le due ideologie sociali della swa rivoluzione: il melting pot dell'inizio del secolo, della generazione non-scolarizzata dei genitori o dei nonni, e il multiculturalismo degli anni settanta e degli anni ottanta.

Né è il riscatto del sentimento o della sentimentalità di stampo puramente tematico. Vi sono testi nella letteratura italo-canadese in cui il personaggio preferisce soprassedere, non nominare la portata politico-sociale della sua traiettoria. V'ittorio Innocente, narratore di Il'bere She bas Gone, terzo volume di una trilogia narrativa di Nino Ricci, si destreggia tra le sue tappe di figlio di emigranti, consumandole una per una con puntualità 
perfino eccessiva, che non lascia margini di manovra, come se lo scopo fosse di liberarsene, di mettersele alle spalle una volta per sempre (il tentativo di fondare una vita senza $i$ genitori si traduce in una impossibile relazione incestuosa con la sorella, il ritorno in Italia viene eseguito direttamente, con tutti i crismi, per essere subito ripudiato). "E il sì e il no, la precisione che le cose prendevano nel mondo ordinario non sarebbero stati importanti. Importa solo il racconto, quel brandello di speranza"6: sono le parole che chiudono il prologo del libro, anticipando lo scioglimento, la soluzione a cui mira, ancora senza saperlo, il protagonista - lo stoicismo dell'artista che trova il suo più alto valore nell'opera, nell'atto stesso del narrare. Ma l'elogio dell'etica affiliativa (piuttosto che filiale) dell'arte, della professione, non impedisce al testo di iscrivere anche il padre, la madre, la sorella e gli altri personaggi italiani, canadesi o italo-canadesi nella storia. Con tutta la sua imperfezione e la sua vaghezza, il linguaggio, dichiara nell'epilogo il narratore, è come il fuoco per gli uomini e le donne delle caverne: anche noi ciò che cerchiamo tramite la letteratura alla fine è "di tenere a bada il buio che avanza" $(320)^{-}$.

Il filone stoico-sentimentale installa il buio e il filo di speranza nel cuore della trama, del libro: li rappresenta. Poiché lo scacco è stato immenso (gli ideali traditi sembravano realizzabili: ci fu un tiepido tentativo di legislazione multiculturalista nel Canada) e chiama in causa protagonisti e scrittori in quanto prole universitaria degli emigranti, le taumaturgie si rifrangono. La solidarietà con cui i personaggi reagiscono agli eventi, la pietas che li spinge a ricordare tale o talaltro parente o amico riproduce in piccolo l'anamnesi, la solidarietà, la pietas insita in ogni opera letteraria. I testi "emotivi" esibiscono un tasso di autoriflessività non meno palpabile di quello dei testi "sperimentali". Nelle poesie di Di Cicco o di Gasparini i genitori o i parenti sono spesso colti nel momento in cui stanno per essere fotografati, forse per l'ultima volta nella loro vita ${ }^{8}$. Menzionando, confessando la propria proclività a documentare, come fa Mary Di Nichele, per esempio, ("Guardo un ragazzo sterminare delle formiche.../ Qui registro come gli insetti per terra/zampettano verso la salvezza”, 141) si enuncia l'sstanza letteraria, e non soltanto morale, a cui obbedisce il testo, si trasmuta il testo in un'opera en abyme. E questo accade, nel filone stoico-sentimentale, anche quando i sentimenti e l'impegno etico sembrano avere il sopravvento, sembrano voler rinnegare la letteratura o, addirittura, la propria letterarietà:

La poesia al massimo mi lascia freddo

mio padre ha subito più

oltraggi di quanti le parole 
potrebbero mai sognarsi

di evocare...

M'immagina infelice

perché scrivo, lo disarma

ancora di più

che dopo tutti yuesti

paesi e tutte queste

sconfitte, anche il suo figlio

più giovane non riesca a sfuggire

Dovrei mollare le poesie

e questa lingua e

far saltare in aria

tutti i caseggiati per cui

ha trasportato cemento

Non ha costruito lui la mia prigione.

Non posso distruggere tutti questi

paesi, Padre

chiedo scusa per questi canti. (D'Agostino 84)

- V'a bene per la problematica dell'emergenza come essa ricorre nella letteratura italo-canadese. Con le letterature emergenti molto ruota-richianamente, andrebbe forse meglio sottolineato- intorno alla convertibilità di poesia e storia. E confondere questa convertibilità con un eccesso di sociologismo — l'accusa spesso rivolta a scrittori e testi italo-canadesiè un segno o di impreparazione o di supponenza critica, se è vero che autorappresentarsi tramite la letteratura, iscriversi negli archivi culturali di una società può creare, può, per adoperare un verbo adesso di moda, "costruire", la storia, così come la storia, la tradizione può creare letteratura. D'accordo. Tu stesso però lasci intendere che grandi barlumi di questa problematica si riscontrano presso la cultura italiana, soprattutto in momenti chiave come l'Ottocento pre-risorgimentale, quando la questione dell'identità nazionale, delle connivenze tra storia e letteratura o tra letteratura e storia è all'ordine del giorno e ispira portavoci illustrissimi. Mutatis mutandis, ci par di capire, la posizione psico-storica degli intellettuali italocanadesi riecheggia yuella degli intellettuali italiani di Foscolo e Leopardi, intrappolati anche loro tra ideali e realtà, tra il desiderio di identita e la raggelante forza maggiore della politica, tra l'ottimismo del cuore e il pessimismo della ragione. E allora la letteratura italo-canadese non ci mette di fronte, anche a sorvolare sulle scorie sociologistiche imputategli, a tematiche anacronistiche, ottocentesche, che l'Italia e le nazioni d'Europa 
hanno già "superato"? Dov'è lo scarto che rende la cultura dell'emigrazione "altra", aggiuntiva qualitativamente rispetto all'Italia? E se non c'è scarto, a che pro postulare l'allargamento dell'italianistica? Criticamente quali benefici se ne ricaverebbe?

- Per cominciare, gli aspetti della cultura italiana che il dossier sull'emigrazione elicita non sono più recepiti dalla cultura italiana e dall'italianistica, oppure sono recepiti debolmente. Mediante la loro ermeneutica dislocata, deterritorializzata, gli emigranti rammentano all'Italia, reinstallandoli nel suo presente, alcuni dei suoi più imprescindibili trascorsi. Riesumata attraverso il filtro che imbastisce l'italianità fuori d'Italia, la storia italiana è pervasa dalla casistica dell'emergenza, è una storia perennemente ossessionata dalla prima e definitiva caduta di Roma, dall'immagine dell'erba che cresce tra le rovine, e perennemente tesa ad esorcizzarne il ricordo, ad immaginarsi rinascimenti e risorgimenti9 ${ }^{9}$ Autori come Foscolo, come Leopardi e, a suo modo, come Manzoni generalizzano il segreto rituale di questa storia, abbinandolo alla parimenti laica liturgia dell'arte. Ne I Sepolcri i custodi delle tombe sono le Muse, l'armonia del cui canto "vince di mille secoli il silenzio" (62). Simile alle lapidi funerarie, la poesia annuncia una morte, l'arvenuto trapasso, il non-essere di qualcuno o di qualche cosa, e lo celebra, lo difende dall'incuria del tempo trasferendolo nell'ultramondo laico della finzione e della scrittura, nel regno della memoria. Che è la fonte di ogni rinnovamento. Nell'arte vita e morte camminano braccio a braccio: una si ricollega all'altra, dialetticamente. Nel convertirsi in storiografia, un testo è insieme pensiero della metamorfosi e dell'utopia e pensiero del ritorno e del realismo, pensiero dell'inizio (di un inizio storico) e pensiero della fine (di una fine storica).

A mio arviso, interpretare la storia italiana a partire dal concetto di emergenza ( e dei suoi corollari) non annulla gli scarti con l'ltalia dell'emigrazione. Il movente storico de I Sepolcri è il decreto delle autorità cisalpine che comandara di seppellire tutti i morti in fosse comuni. Per Foscolo e per Leopardi, il Leopardi delle canzoni, la sepoltura assicura differenza gerarchica in aggiunta alla differenza identitaria. La tomba assurge a monumento pubblico per meritocrazia, se le spoglie che in essa riposano sono quelle di un individuo di chiara fama. Il cimitero prelude al pantheon nazionale, ai mausolei delle tombe eccellenti. L'na inderogabile necessità per chi, come Foscolo e Leopardi, credera che l'insipienza politica degli Italiani fosse dovuta ad un deficit di autostima. La visita a Santa Croce, alle tombe dei Petrarca, dei Galileo, degli Alfieri, conforta i propositi battaglieri di Jacopo Ortis, il personaggio del romanzo foscoliano; analogo effetto ortatorio spera che abbiano le sue evocazioni degli stessi spiriti, dei lari 
della storia italiana l'io delle canzoni leopardiane. Nella letteratura italocanadese, la memoria non è, e non può esserlo, al servizio dello stato, della nazione, nascente o no. Il testo, se mai, funge da rimembranza del gruppo, che non ha velleità totalizzanti, non pretende di coincidere con la nazione, mira semplicemente al riconoscimento di sé in yuanto gruppo.

Tra l'altro il passato che Foscolo e l copardi recuperano è quello agiografico e renerabile dei grandi ingegni, quello trionfale di Firenze, Roma, Milano, Venezia, Genova, forse Napoli. Il passato dei Paci, dei Ricci, dei Di Cicco, dei Di Michele è yuello, molto più sommesso, mai garantito (e dunque "aggiuntivo") della provincia, dei villaggi, della massa contadina post-risorgimentale, sia che la si concepisca come una folla che ha votato con i piedi, andandosene, o come una folla travolta dalla frumana dell'economia (l'emigrazione obbedisce a certi ritmi, ad una sistole e diastole sociale, al bisogno di manodopera a basso costo di alcuni pacsi e al bisogno di ridurre la sorrappopolazione da parte dei paesi con scarse risorse, esportatori di lavoratori). Pur mantenendo intatti gli agganci tra arte e storia o tra arte e memoria, i narratori dei romanzi o delle poesie italo-canadesi ne democratizzano la portata. L'atto dell'iscrivere ristabilisce una continuità storica, riafferma la paternità o la maternità dei genitori, e riconcilia con essi e con la loro storia sulla base, ripeto, di una comune umanità. Nel recepire l'antica esortazione di Antigone a seppellire e commemorare, i personaggi e gli scrittori e le scrittrici della letteratura italocanadese si fanno figli-fratelli e figlie-sorelle dei loro padri e delle loro madri.

Con questo non voglio insinuare che l'incongruenza italo-canadese sta in una qualche inedita miscelazione del sublime patriottico di Foscolo e del primo Leopardi con la religiosità o la poetica degli umili di Manzoni, per quanto accattivante (e per me lo è) possa essere un tale sospetto. lo scarto che separa la cultura italo-canadese dalla cultura italiana d'Italia viene dal contesto. Per tener adeguato conto dell'emigrazione, ho detto, si dere cambiare lo spazio-tempo dell'italianistica. E ho detto pure che un tale spaziotempo - lo spazio-tempo dell'italianità- è per natura eterogeneo, dinamico, una geografia-cronologia da esplorare comparativamente. Mettendo in primo piano le cesure, i periodi che progetta un yualche emergere (o un qualche ri-emergere), si può far vedere che la critica italo-canadese troverebbe anche in Italia e nelle cultura italiana attrezzatura metodologica onde affilare e arricchire la propria utensileria, che ripensare il cordone ombilicale sbiadito o abbandonato o sparito è per la critica italo-canadese un passaggio obbligato, cosi come per la critica italiana è un passaggio obbligato prendere atto della presenza di un'italianità fuori d'Italia e misurarsi seria-mente con i suoi cronotopi storici e/o culturali. Bene. I 
Foscolo, i Manzoni e gli ottocentisti italiani vivono all'epoca del nazionalismo romantico o post-romantico, le comunità degli emigranti italocanadesi, non va dimenticato, non dispongono di una precisa identità giuridica - attribuibile, oggi, ancora solo a chi può reclamare dominio su un territorio- e con la nazione canadese o italiana intrattengono i rapporti che la loro epoca - l'epoca della globalizzazione-consente. Per cui le tematiche che emana-no dai paralleli tra le due fasi germinative sono lungi dall'essere superate. Ma io andrei ancora più in là. Si può, senza esagerare, asserire che alcune delle questioni che gli scrittori italo-canadesi hanno doruto meditare, sia pure quella apparentemente anacronistica che pertiene all'utilizzo del passato in un paese di immigranti, multi-culturale e multi-etnico, anticipano un futuro per l'Italia ancora storicamente dietro l'angolo ma che sicuramente avrà le sue ricadute critiche.

Infine, per procedere al versante spaziale, il Canada è un altro luogo, con tutt'altra configurazione geografica e culturale. Il che significa anche questo è un fattore da non dimenticare e da non trivializzare - che lo scarto dell'emigrazione, lo scarto che ci riconsegna l'italianità, italianità "italiana" compresa, in un'altra luce, lo danno i contenuti, non i processi, che invece si corrispondono. Ecco, ognuno degli argomenti che ho sommariamente sintetizzato - le connivenze tra letteratura e memoria culturale, tra memoria culturale e identità nazionale, tra presente e passato, tra localismo e cosmopolitismo, tra emigrazione e postcolonialismo rispunta in altre sedi, in quelle sedi critiche più transitate oggi dagli studi letterari. Ognuno, per vie dirette o per vie traverse, redime l'italianistica, la riporta in carreggiata, le restituisce una qualche misura di paradigmaticità. Ma forse la sfida ultima che la cultura dell'emigrazione riserva all'italianistica consiste in questo: come reinsediare, senza banalizzarla, la questione del contenuto nel dibattito sulla letteratura. Per la critica le conseguenze sono immense. Anche per abbozzarne un primo, schematico elenco, dorrei aprire un nuovo e lunghissimo discorso. Sarà per un'altra volta.

\section{Carleton L'niversity}

\section{NOTE}

1 Quale sia l'atruale capitale simbolico della letteratura italiana nel Nord America lo indica bene Bernheimer (44-45): " $[1]$ t shoould be acknowledged that minority literatures also exist within Europe. Eurocentricity in practice entails a focus on English, French. German and Spanish literatures. Even Italian literature, with the exception of Dante, is often marginalized."

2 Per Riccardo Petrella $(1991,1992)$ la situazione attuale ha il suo parallelo storico nella lega anseatica. 
3 Va da sé, ma è meglio essere espliciti, a scanso di equivoci, che gli italianisti di origine non-italiana arrebbero un loro importantissimo ruoke in una rassegna dell'italianistica canadese e la loro presenza sarebbe da includere in ogni calcolo finale. (Qui ho assunto il punto di vista "interno", di chi ha vissuto l'emigrazione. Non è detto che sia la sola ottica accettabile, o che degli emigranti italiani in Canada possa parlare solo un altro emigrante jtaliano, cosi come non é detto che per parlare degli italianisti di origine non italiana sia necessario avere un yualche particolare passaporto. Il punto di vista interno, "autobiografico", pero, è stato finora, ingiustamente, yuello più spesso inibito (per ragioni che hanno a che fare con il preponderante prestigio dell'oggettività, dell'impersonalità, della scientificita presso la critica moderna), e quindi, oggi come oggi, è ciò che più urge recuperare.

4 Menzionerei Enilio Franzina, Armando Gnisci e il gruppo intorno alla rirista Altreitalie.

5 "Fàtica" nel noto modello della comunicazione verbale elaborato da Roman Jakobson è quella funzione della lingua - re ne sono sei - che dà risalto al canale tramite cui arriene la trasmissione del messaggio. Si riconnette dunyue al bisogno, da parte del mittente o del destinatario di prolungare la comunicazione, o comunque di far sì che non sia interrotta. Jakobson, come spiega nel suo saggio (301), mutua il termine dall'antropologo Bronislaw Maliknowski, per il quale anche i miti e la narrativa folklorica poterano svolgere una funzione fatica. La genealogia è oggi di suggestiva risonanza. Perché ad ascoltare alcuni teorici del nazionalismo, Benedict Anderson in primis, la "comunità immaginaria" implicita nell'idea di nazione nasce grazie alla mediazione della lingua scritta e di certi generi letterari a larga diffusione. La letteratura dell'epoca moderna, una letteratura che beneficia dell'invenzione della stampa, che riesce a circolare dove non giunge la comunicazione orale, a tenere in contatto persone che non si redono e non possono parlarsi, intensifica il grado di coesione di un determinato gruppo sociale. La poesia, il teatro, la narrativa emergente sono i testi della modernità in cui l'aspetto fatico della letteratura viene potenziato al massimo, viene ad aggiungersi a tutte le altre questioni di forma o di contenuto che gravitano intorno alla letteratura, e di cui la critica non può mai fare a meno. Nell'ambito italo-canadese italofono il contatto lo garantisce la lingua; in quella anglofona o francofona, la dimensione tematica, le sue tensioni con l'inglese o il francese che la veicolano.

${ }^{6}$ la pagina non è numerata.

7 La traduzione dei brani di Ricci è mia.

8 Di Di Cicco si redano i seguenti versi:" cè questo uomo la cui capigliatura splenderà come/le foglie di olivo a mezzogiono; ì quest'uomo che siederà/a mezzogiorno sul prato daranti alla casa/dopo la quinta emorragia/posando senza saperlo per l'ultima foto...(19-9:11, traduzione mia); "C'c una casa non troppo distante/anche se sembrano miglia, una casa di rose, con un/recchio sul prato davanti, che si arriccia i baffi/per la sua ultima foto..."(1998:107). Di Gasparini: "IE mentre il resto della famiglia giocara/ a carte o ascoltava musica italiana/io ne 
studiavo il volto rugoso, sereno/e l'amavo. L'na volta venne fotografato senza che neppure/lo sapesse." (1998:174).

${ }^{9}$ Non è senza interesse che con la creazione dello stato italiano si passi, nel periodo di più drammatica riverberazione di questo secolo, da un "risorginento" ad una "resistenza". La Resistenza degli anni quaranta rintuzza l'erentualità di una nuova morte sociale, ripete sotto nuove spoglie - dall'altro lato, dal lato di un risorgimento avvenuto - il ciclo storico del rinascere e del morire. Forse è questo il futuro macrostorico dell'Italia “italiana”. Direttamente l'idea di risorgimento sembrerebbe oggi riproponibile solo dagli emigranti italiani all'estero e dagli immigrati stranieri in Italia. $\Lambda$ meno che l'Italia non rivisiti il proprio passato pre-ottocentesco, non arrivi a considerarlo inestricabilmente intrecciato con quello degli immigrati, e quindi a concepire il suo prossimo futuro come un "ritorno", una qualche "rinascita". Il Risorgimento degli emigranti, invece, resta doppio; è un Risorgimento in un altro luogo, come quello degli immigrati in Italia.

\section{OPERE CITATE}

Anabile, George, "Divario generazionale". Traduzione di L.eonardo Buonomo. In Loriggio, 35-42.

Anderson, Benedict, Imagined Comminities. Reflections on the Origins and Spread of Nationalism. Edizione riveduta. London:Verso, 1991.

Bernheimer, Charles et alii, "The Bernheimer Report", in Comparative I iterature in the Age of Multionlturalism. Ed. Charles Bernheimer. Baltimore: Johns Hopkins University Press, 1994.

D’Agostino, Saro, "Canti d'immigrati". Traduzione di Leonardo Buonomo. In Loriggio, $82-85$

De Luca Calce, Fiorella, Toni. Toronto: Guernica, 1989.

Di Cicco, Pier Giorgio, "The Man Called Beppino", in The Tough Romance. Toronto: McClelland and Stewart, 1979. 11.

"Ricordando Baltimore, Arezzo". Traduzione di William Anselmi. In Loriggio, 107-108.

"America". Tracluzione di Wrilliam Anselmi. In Loriggio, 109-110.

Di Michele, Mary, "Mimosa". Traduzione di Cristina Trevisan. In Loriggio, 132135.

" "I nomi dei morti". Traduzione di Cristina Trevisan. In Loriggio, 141.

Evans, Peter, "The Eclipse of the State? Reflections on Stateness in an Era of Globalization". II"orld Politics 50 (1997): 62-87.

Foscolo, Ugo "Dei Sepolcri”, in Le Poesie. Milano: Garzanti, 1974, $51-65$.

Franzina, Emilio, Gli italiani al Nuovo Mondo 1492-1942. Milano: Mondadori, 1995. Galli della Loggia, Ernesto, L'identità italianı. Bologna: Il Mulino, 1998.

Gasparini, Len, "La fotografia di mio nonno che legge Dante". Traduzione di Leonardo Buonomo. In Loriggio, 174.

Gnisci, Armando, Ia letteratura italiana della migrazione. Roma: Lilith Edizioni, 1998 Huntington, Samuel P. The Clash of Cirilizations. New York: Simon and Schuster, 1996 
Hirst, Paul e Thompson, Grahame, Globulizution in (Ouestion. Cambridge: Polity Press, 1996.

Jakobson, Roman, "Linguistics and Poetics", in Lssals on the I angunge of I iterature. Eds. Sermour Chatman and Samuel R. Levin. Boston: Houghton Mlifflin Co: 1967. 296-322.

Jones, R.J. Barry; The I"brld Tumed L pside Donn! Cilobulization and the Future of the Stute.Manchester: Manchester L niverity Press, $20(1)(0$.

Loriggio, Francesco, a cura di, L isltra storia. Antologia della letteratura italo-canadese. Vibo V'alentia: Monteleone, 1998.

Maliknowski, Bronislaw; "The Problem of Meaning in Primitive Languages", in

The . Yeaning of Meaning. Eds. C. K. ()gden and I.A. Richards. London: Kegan Paul, 1936. 296-336.

Paci, Frank, "Da L'nder The Bridge". Traduzione di Leonardo Buonomo. In loriggio, 282-296

Petrella, Riccardo, "World City-States of the Future," Nen' Perspectives Quarterly, (Fall 1991): 59-64

"A New Hanseatic League?" Nen' York Times, February 23, 1991, E3

Ricci, Nino, I'bere She Has Gone. Toronto: McClelland and Stewart, 1997.

Smith, Anthony D. Nations and Nationalism in a Global Era. London : Polity Press, 1996.

Sterenson, Nick, "Globalization, National Cultures and Cultural Citizenship", The Saciological Quarterh', 38, 1 (1997): 41-66.

V'iscusi, Robert Astoria. Toronto: Guernica, 1994. 